\title{
Normalized Hand Grip and Back Muscle Strength as Risk Factors for Incident Type 2 Diabetes Mellitus: 16 Years of Follow-Up in a Population-Based Cohort Study
}

This article was published in the following Dove Press journal: Diabetes, Metabolic Syndrome and Obesity: Targets and Therapy

\author{
Yoo-Jeong Jeon (iD) \\ Seung Ku Lee 2,3 \\ Chol Shin (iD) ${ }^{2-5}$ \\ 'Department of Sports \& Health Science, \\ Hanbat National University, Daejeon, \\ Korea; ${ }^{2}$ Institute of Human Genomic \\ Study, College of Medicine, Korea \\ University Ansan Hospital, Ansan-si, \\ Gyeonggi-do, Republic of Korea; ${ }^{3}$ College \\ of Medicine, Korea University, Seoul, \\ Republic of Korea; ${ }^{4}$ Department of \\ Pulmonary, Sleep and Critical Care \\ Medicine, College of Medicine, Korea \\ University Ansan Hospital, Ansan-si, \\ Gyeonggi-do, Republic of Korea; \\ ${ }^{5}$ Transdisciplinary Major in Learning \\ Health Systems, Department of \\ Healthcare, Sciences, Graduate School, \\ Korea University, Seoul, Republic of \\ Korea
}

Purpose: Muscle strength is associated with type 2 diabetes mellitus (T2DM). However, it is controversial whether muscle strength and normalized muscle strength is a risk factor for T2DM. Moreover, the relationship of back muscle strength (BMS) and incident T2DM has not been reported. In this study, we investigated the relationship between HGS, BMS, normalized HGS and BMS, and incident T2DM.

Methods: A total of 2699 non-diabetes subjects aged 40-69 years (1313 women and 1386 men) in the Korean Genome and Epidemiology Study (KoGES) Ansan cohort were followed for 16 years. At the baseline and biennial follow-up visits, fasting glucose, postprandial 2-h glucose, clinical examinations, HGS, and BMS were measured by trained interviewers and examiners. HGS and BMS were measured at baseline. The relationships between incident T2DM, HGS, BMS, and normalized HGS and BMS were estimated using Cox proportional hazard regression models after adjusting for the confounding factors.

Results: HGS and BMS were not associated with incident T2DM in multivariate analysis. However, the hazard ratio (HR) per one standard deviation (SD) increase in the body mass index (BMI), waist circumference (WC), waist-hip ratio (WHR)-normalized HGS, and BMS was associated with a lower risk for incident T2DM in both women and men after adjusting for the confounding factors ( $\mathrm{HR}=0.842-0.880$-fold for women, $\mathrm{p} \leq 0.015$; HR $=0.887-0.903$ fold for men, $\mathrm{p} \leq 0.024)$. In the sub-analysis of menopause status, the HR per one SD increase in BMI, weight ${ }^{2 / 3}, \mathrm{WC}$, and WHR-normalized HGS was associated with a lower risk for incident T2DM in both pre- and post-menopausal women after adjusting for the confounding factors $(\mathrm{HR}=0.860-0.820$-fold for premenopausal, $\mathrm{P} \leq 0.006 ; \mathrm{HR}=0.900-0.867$-fold for postmenopausal, $\mathrm{p} \leq 0.024$ ). Additionally, we confirmed that the quartile group with higher muscle strength was associated with a lower risk for incident T2DM.

Conclusion: The present study suggested that normalized HGS and BMS were associated with a lower risk for the future development of T2DM. Moreover, weak muscle strength in premenopausal women may be the cause of T2DM. Further research is needed to determine whether efforts to improve muscle strength, such as exercise can reduce the risk of T2DM. Keywords: incident type 2 diabetes mellitus, hand grip strength, back strength

\section{Introduction}

Type 2 diabetes mellitus (T2DM) is a chronic disease requiring medical treatment and self-management education. ${ }^{1}$ Patients must make continuous efforts to prevent complications because diabetes can lead to microvascular complications such as
Correspondence: Chol Shin

Department of Pulmonary, Sleep and

Medicine, Korea University Ansan

Hospital, 123 Jeokgeum-ro, Danwon-gu,

Gyeonggi-do, Ansan-si, 15355, Republic of

Korea

Tel +82-3I-4I 2-5603

Email chol-shin@hanmail.net
Diabetes, Metabolic Syndrome and Obesity: Targets and Therapy 202I:14 74I-750 
cardiovascular disease, stroke, and chronic kidney disease, which contribute to mortality. ${ }^{2}$ Therefore, the early prevention and management of T2DM, which underlies various chronic diseases, can improve national health and reduce the burden on the national economy due to medical expenses. $^{3}$

Muscle strength is a simple and reliable metric used to evaluate muscle function. ${ }^{4}$ Although the relationship between muscle mass and muscle strength are interdependent, muscle strength may be considered an important disease predictor because muscle strength decreases $21 \%$ to $40 \%$ faster than muscle mass. ${ }^{5}$

Previous studies indicated that hand grip strength (HGS) was associated with T2DM. ${ }^{6-8}$ However, there is also evidence that HGS is not related to T2DM. ${ }^{9-11}$ Muscle strength shows ethnic differences. The prevalence of weak strength in non-Hispanic Asians is $10 \%$ higher than in non-Hispanic white and non-Hispanic black people. ${ }^{12}$ Moreover, lifestyle and physical health at young ages determine the rate of decline in muscle strength in old age. ${ }^{13}$ Therefore, the study results will be inconsistent if the above problems are not addressed.

Body composition and body size were highly correlated with muscle strength, ${ }^{14}$ indicating that large body sizes may have larger muscle mass. Therefore, body composition and body size-independent measurements for application of muscle strength is important. The use of a normalized method is recommended when investigating the relationship between disease states and mobility. ${ }^{15,16}$ Body mass index (BMI), weight, weight ${ }^{2 / 3}$, waist circumference (WC), and the waisthip ratio (WHR) are well-known factors used for normalizing muscle strength. ${ }^{9,17-20}$ There is little research on back muscle strength (BMS). Back strength was significantly correlated with HGS and total body muscle mass. Therefore, its correlation with HGS needs to be considered. ${ }^{21}$ BMS was associated with insulin, the Homeostasis Model Assessment (HOMA) of insulin resistance (HOMA-IR), and the HOMA of $\beta$-cell function (HOMA-B). ${ }^{22}$ However, the relationship between BMS and incident T2DM has not been reported.

Therefore, this study aimed to evaluate the relationship between HGS, BMS, BMI, weight, weight ${ }^{2 / 3}$, WC, WHRnormalized HGS and BMS, and incident T2DM in a 16year, longitudinal large cohort.

\section{Materials and Methods}

\section{Study Subjects and Population}

The subjects were selected from the participants in the Korean Genome and Epidemiology Study (KoGES), an ongoing prospective population-based study in an Ansan cohort. The Ansan cohort was initiated in 2001 and has been followed biennially. At baseline, the initial cohort of 5012 subjects aged 40 to 69 years was randomly recruited from the urban community of Ansan (2518 men and 2494 women). The data collected from the cohort included questionnaires, anthropometric measurements, blood tests, and clinical examinations by trained interviewers and examiners. The follow-up rate at the ninth examination was $64.2 \%$.

In the present study, 883 subjects were excluded at baseline [muscle strength $(\mathrm{N}=332), \mathrm{BMI}(\mathrm{N}=1)$, WC $(\mathrm{N}=7)$, and T2DM $(\mathrm{N}=544)$ ] (Figure 1). During the 16-year study period, 2699 out of 4129 subjects were lost to follow-up due to death $(\mathrm{N}=282)$ and lack of participation $(\mathrm{N}=1148)$. Finally, 2699 subjects remained eligible for this investigation. This study was approved by the Institutional Review Board of Korea University Ansan Hospital, and written informed consent was provided by all study subjects, and was conducted in accordance with the Declaration of Helsinki.

\section{Muscle Strength Measurements}

HGS and BMS were measured at baseline using digital dynamometers (Grip-D T.K.K.5401 and T.K.K.5102, TAKEI Science Instruments Co., Ltd, Nigata, Japan). The maximum HGS was measured after the grip was maintained at $15^{\circ}$ from hip flexion and measured three times in each hand with a one-min rest interval. To measure BMS, the participants stood upright and were positioned with their hands on the knob. The pulling force was measured three times by straightening the waist, with a one-min rest interval. The average muscle strength, BMI, weight, weight ${ }^{2 / 3}$, WC, and WHR-normalized muscle strength was used in this study because normalized

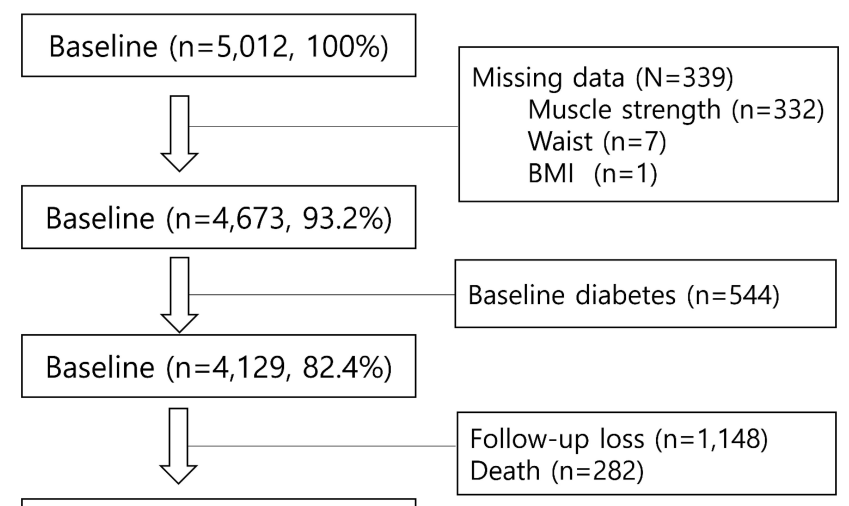

Follow-up ( $n=2,699,53.9 \%)$

Figure I Participant flowchart. 
muscle strength has been proposed as an alternative and more precise diagnostic tool for assessing the muscular strength of overweight individuals. WC and WHR are highly correlated with BMI and are known as risk factors for diabetes. ${ }^{23}$ All possible combinations were assessed.

\section{Definition of Type 2 Diabetes Mellitus and Hypertension}

We used the diagnostic criteria for diabetes and hypertension proposed by the World Health Organization. ${ }^{24,25}$ All blood samples were collected after 8-12 hours of fasting. The serum was separated by centrifugation at $3000 \mathrm{rpm}$ for $10 \mathrm{~min}$ at room temperature (RT) for the analyses after clotting for 30 min at RT. In addition to the fasting levels of glucose and insulin, glucose and insulin levels were also measured one and two $\mathrm{h}$ after the ingestion of $75 \mathrm{~g}$ of glucose. T2DM was defined as a fasting plasma glucose concentration of $\geq$ $126 \mathrm{mg} / \mathrm{dL}$, or a postprandial 2-h glucose concentration of $\geq$ $200 \mathrm{mg} / \mathrm{dL}$, or current treatment with oral anti-diabetic drugs or insulin. During the follow-up period, glucose levels investigated using the same method and defined incident T2DM. Before measuring blood pressure (BP), smoking or coffee consumption coffee was not allowed for at least 30 minutes. BP was measured by trained examiners using an appropriately sized cuff and a mercury sphygmomanometer. The first and the fifth phases of Korotkoff sounds were used for systolic and diastolic BP, respectively. BP measurements were made after a rest period of at least five min in the sitting position, and repeated twice, with a 30 -s recovery period. The average of the two readings was used for the analysis. Hypertension was defined as systolic blood pressure (SBP) of at least 140 $\mathrm{mmHg}$ or diastolic blood pressure (DBP) of at least 90 $\mathrm{mmHg}$, or treatment with antihypertensive medication.

\section{Covariates}

Study subjects completed questionnaires administered by a trained interviewer, which included questions on demographic information, current and past medical conditions, family history of diseases, and lifestyle. At baseline, a family history of diabetes (FHD) was defined as a positive parental history of diabetes. Leisure-time physical activity was calculated using questionnaires, which included questions about the type of physical activity, frequency (times per week), and duration (in minutes). Metabolic equivalent (MET) values were assigned to each sports activity based on a compendium of physical activities. Alcohol consumption was calculated using questionnaires including the type of drinks (beer, wine, hard liquor, and three types of traditional drinks, including soju, chungju, and makgeolli), amount, and frequency (times per week). Smoking status was categorized as never, past, or current. Menopause was categorized as premenopausal and postmenopausal. BMI was calculated as weight divided by height squared $\left(\mathrm{kg} / \mathrm{m}^{2}\right)$. Triglycerides, high-density lipoprotein (HDL)-cholesterol, fasting glucose levels, and postprandial 2-h glucose were measured using an ADVIA 1650 Auto Analyzer (Siemens Medical Solutions, Tarrytown, NY, USA). Energy consumption (kcal/day) for the day was calculated using a food frequency questionnaire.

\section{Statistical Analysis}

The baseline characteristics are expressed as means and standard deviations for the continuous variables and as percentages for the categorical variables. Differences in the examinations were determined using generalized linear models and chisquared tests. To estimate the risk of developing T2DM, we applied Cox proportional hazards regression models (HRs), 95\% confidence intervals (CI), and P-values where the HR represents one standard deviation (SD) in muscle strength and normalized muscle strength in the risk of developing T2DM after adjusting for the confounding factors. Additionally, muscle strength was grouped into quartiles according to muscle strength by sex. In each case, the lowest quartile group was fixed as the reference group. The potential confounding variables $^{26,27}$ adjusted for in the multivariate models were age, FHD (yes, no), exercise (METs), alcohol consumption (g/day), smoking status (never smoker, ex-smoker and current smoker), job (housekeeper, white-collar, and blue-collar), triglycerides, HDL-cholesterol, fasting glucose, hypertension, cardiovascular diseases, and energy consumption from the food frequency questionnaire (FFQ) for men at baseline, and adjusted for menopause in women at baseline in addition to the adjustments made for men. For sensitivity analyses, we calculated the HR of the normalized HGS and BMS for incident T2DM in the second, sixth, and ninth follow-up periods. A two-tailed $\mathrm{P}<0.05$ was considered statistically significant. All statistical analyses were performed using SAS statistical software (SAS 9.4, SAS Institute, Cary, NC, USA).

\section{Results}

\section{Characteristics of the Study Subjects}

The baseline characteristics of the study subjects according to sex are shown in Table 1. This study included 1313 women and 1386 men. The mean age (48.1 \pm 7.0 for women and 48.0 \pm 6.8 for men), BMI (24.6 \pm 3.0 for women and $24.7 \pm 2.7$ for 
Table I Baseline Characteristics According to Sex

\begin{tabular}{|c|c|c|c|c|}
\hline Variables & & Women $(n=|3| 3)$ & Men $(n=1386)$ & P-value* \\
\hline Age (years) & & $48.1 \pm 7.0$ & $48.0 \pm 6.8$ & 0.675 \\
\hline Body mass index $\left(\mathrm{kg} / \mathrm{m}^{2}\right)$ & & $24.6 \pm 3.0$ & $24.7 \pm 2.7$ & 0.806 \\
\hline Weight (kg) & & $59.4 \pm 7.7$ & $69.7 \pm 9.0$ & $<0.001$ \\
\hline Waist circumference $(\mathrm{cm})$ & & $76.5 \pm 7.5$ & $83.3 \pm 7.2$ & $<0.001$ \\
\hline Height $(\mathrm{cm})$ & & $|55.3 \pm 5|$. & $168.0 \pm 5.7$ & $<0.001$ \\
\hline Alcohol consumption (g/day) & & $1.6 \pm 5.8$ & $17.9 \pm 25.5$ & $<0.001$ \\
\hline Exercise (Met) & & $123.8 \pm 196.5$ & $119.8 \pm 195.9$ & 0.599 \\
\hline Fasting glucose $(\mathrm{mg} / \mathrm{dL})$ & & $80.8 \pm 7.6$ & $86.5 \pm 9.4$ & $<0.001$ \\
\hline HDL-cholesterol (mg/dL) & & $52.1 \pm 11.9$ & $46.5 \pm 10.4$ & $<0.001$ \\
\hline Triglycerides (mg/dL) & & $124.7 \pm 78.4$ & $166.8 \pm 108.4$ & 0.002 \\
\hline Energy consumption (kcal/day) & & $|740.9 \pm 49| .3$ & $2017.6 \pm 510.8$ & $<0.001$ \\
\hline Family history of diabetes ( $\mathrm{n}(\%))$ & & $186(14.2 \%)$ & $161(11.6 \%)$ & 0.048 \\
\hline Hypertension (n (\%)) & & $221(16.8 \%)$ & $338(24.4 \%)$ & $<0.001$ \\
\hline Cardiovascular diseases (n (\%)) & & 14 (I.1\%) & $21(1.5 \%)$ & 0.303 \\
\hline Menopause (n (\%)) & & $262(20.0 \%)$ & - & - \\
\hline Ex- or current smoker $(\mathrm{n}(\%))$ & & 44 (3.4\%) & $1075(77.6 \%)$ & $<0.001$ \\
\hline \multirow[t]{3}{*}{ Job (n (\%)) } & Housekeeper & $864(65.8 \%)$ & $2(0.1 \%)$ & $<0.001$ \\
\hline & White collar & $53(4.0 \%)$ & 325 (23.4\%) & \\
\hline & Blue collar & $396(30.2 \%)$ & $1059(76.4 \%)$ & \\
\hline Hand grip strength (kg) & & $21.8 \pm 3.9$ & $35.5 \pm 5.6$ & $<0.001$ \\
\hline Back muscle strength $(\mathrm{kg})$ & & $42.8 \pm 12.3$ & $84.2 \pm 19.2$ & $<0.001$ \\
\hline
\end{tabular}

Note: *Statistical significance for frequency was analyzed by the Chi-squared test and the continuous variables were analyzed by the $t$-test.

men), exercise, and cardiovascular disease (CVD) status were not different between women and men. However, weight, WC, height, alcohol consumption, fasting glucose, triglycerides, and energy consumption levels were significantly higher in men than in women $(\mathrm{P}<0.05)$. HDLcholesterol was significantly higher in women than in men $(\mathrm{P}<0.05)$. Women were significantly more likely to have an FHD than men $(\mathrm{P}<0.05)$. The men were significantly more likely to be ex- or current smokers and have hypertension than the women $(77.6 \%$ vs $3.4 \%$ and $24.4 \%$ vs $16.8 \%$, respectively) $(\mathrm{P}<0.05)$. Most women were housekeeper (65.8\%) followed by blue-collar workers (30.2\%), whereas most men were blue-collar workers $(76.4 \%)$, followed whitecollar workers $(23.4 \%)(\mathrm{P}<0.05)$. Twenty percent of the women had experienced menopause.

The baseline characteristics of the study subjects according to non-diabetes, incident diabetes, and sex are shown in Table 2. The mean age, BMI, weight, WC, fasting glucose, triglycerides, HDL-cholesterol, FHD, and hypertension were significantly different between women and men. The mean age, BMI, weight, WC, fasting glucose, and triglyceride levels were higher in patients with incident diabetes $(\mathrm{P}<0.001)$. An FHD and hypertension were found in higher frequency in patients with incident diabetes $(\mathrm{P}<0.05)$. HDL-cholesterol was more prevalent in patients without diabetes $(\mathrm{P}<0.001)$. Height, job, and menopause were significantly different in women compared to men. The non-diabetes patients were taller $(\mathrm{P}=$ 0.002). A higher frequency of people employed as housekeepers was seen in patients with incident diabetes $(\mathrm{P}=0.044)$. Menopause was present in a higher frequency in patients with incident diabetes $(\mathrm{P}=0.001)$. Cardiovascular diseases were present in high frequencies in patients with incident diabetes $(P=0.036)$. Alcohol consumption, exercise (MET), energy consumption, and ex- and current smoking status were not associated with incident diabetes.

\section{Difference in Hand Grip and Back Muscle Strength, and Normalized Muscle Strength According to Non-Diabetes and Incident Diabetes}

HGS, BMS, and normalized muscle strength according to non-T2DM, incident T2DM, and sex are shown in Table 3. The HGS was less in patients with incident T2DM $(\mathrm{P}<$ 0.05). However, BMS was not associated with incident T2DM. Interestingly, BMI, weight, weight ${ }^{2 / 3}$, WC and WHR-normalized in both HGS and BMS were lower in incident T2DM than non-diabetes. 
Table 2 Baseline Characteristics According to Non-Diabetes and Incident Diabetes

\begin{tabular}{|c|c|c|c|c|c|c|c|}
\hline \multirow[t]{3}{*}{ Variables } & & \multicolumn{2}{|c|}{ Women $(n=1313)$} & \multirow[t]{3}{*}{ P-value* } & \multicolumn{2}{|l|}{ Men $(n=1386)$} & \multirow[t]{3}{*}{ P-value* } \\
\hline & & Non-Diabetes & Incident Diabetes & & Non-Diabetes & Incident Diabetes & \\
\hline & & $(n=887)$ & $(n=426)$ & & $(n=836)$ & $(n=550)$ & \\
\hline Age (years) & & $47.0 \pm 6.4$ & $50.3 \pm 7.6$ & $<0.001$ & $47.0 \pm 6.1$ & $49.5 \pm 7.5$ & $<0.001$ \\
\hline Body mass index $\left(\mathrm{kg} / \mathrm{m}^{2}\right)$ & & $24.2 \pm 2.9$ & $25.6 \pm 3.0$ & $<0.001$ & $24.3 \pm 2.6$ & $25.2 \pm 2.7$ & $<0.001$ \\
\hline Weight (kg) & & $58.6 \pm 7.4$ & $61.3 \pm 7.9$ & $<0.001$ & $68.9 \pm 8.8$ & $71.0 \pm 9.2$ & $<0.001$ \\
\hline Waist circumference $(\mathrm{cm})$ & & $75.0 \pm 7.1$ & $79.5 \pm 7.5$ & $<0.001$ & $82.0 \pm 6.9$ & $85.2 \pm 7.2$ & $<0.001$ \\
\hline Height $(\mathrm{cm})$ & & $155.6 \pm 5.0$ & $154.7 \pm 5.2$ & 0.002 & $168.2 \pm 5.5$ & $167.7 \pm 5.8$ & 0.115 \\
\hline Alcohol consumption (g/day) & & $1.6 \pm 5.6$ & $1.7 \pm 6.3$ & 0.730 & $17.1 \pm 25.3$ & $19.0 \pm 25.8$ & 0.174 \\
\hline Exercise (Met) & & $125.7 \pm 196.7$ & $120.0 \pm 196.1$ & 0.623 & $124.7 \pm 190.8$ & $112.5 \pm 203.5$ & 0.259 \\
\hline Fasting glucose (mg/dL) & & $79.6 \pm 6.6$ & $83.4 \pm 8.9$ & $<0.001$ & $84.4 \pm 8.1$ & $89.6 \pm 10.3$ & $<0.001$ \\
\hline HDL-cholesterol (mg/dL) & & $53.0 \pm 11.8$ & $50.1 \pm 11.7$ & $<0.001$ & $47.4 \pm 10.7$ & $45.3 \pm 9.6$ & $<0.001$ \\
\hline Triglycerides (mg/dL) & & $111.2 \pm 68.6$ & $152.8 \pm 89.3$ & $<0.001$ & $148.9 \pm 87.8$ & $194.0 \pm 129.1$ & $<0.001$ \\
\hline Energy consumption (kcal/day) & & $1742.5 \pm 496.0$ & $1737.6 \pm 481.9$ & 0.866 & $2019.4 \pm 501.6$ & $2014.9 \pm 525.0$ & 0.875 \\
\hline Family history of diabetes ( $\mathrm{n}(\%)$ ) & & $113(12.7 \%)$ & $73(17.1 \%)$ & 0.032 & 81 (9.7\%) & $80(14.5 \%)$ & 0.006 \\
\hline Hypertension (n (\%)) & & $120(13.5 \%)$ & 101 (23.7\%) & $<0.001$ & $162(19.4 \%)$ & $176(32.0 \%)$ & $<0.001$ \\
\hline Cardiovascular diseases (n (\%)) & & $9(1.0 \%)$ & $5(1.2 \%)$ & 0.793 & $8(1.0 \%)$ & $13(2.4 \%)$ & 0.036 \\
\hline Menopause (n (\%)) & & $155(17.5 \%)$ & 107 (25.1\%) & 0.001 & & & \\
\hline Ex- and current smoker (n (\%)) & & $26(2.9 \%)$ & $18(4.2 \%)$ & 0.223 & $646(77.3 \%)$ & $429(78.0 \%)$ & 0.751 \\
\hline \multirow[t]{3}{*}{ Job (n (\%)) } & Housekeeper & $569(64.1 \%)$ & $295(69.2 \%)$ & 0.044 & - & $2(0.4 \%)$ & 0.166 \\
\hline & White collar & $43(4.8 \%)$ & $10(2.3 \%)$ & & $202(24.2 \%)$ & $123(22.4 \%)$ & \\
\hline & Blue collar & $275(31.0 \%)$ & $|2|(28.4 \%)$ & & $634(75.8 \%)$ & $425(77.3 \%)$ & \\
\hline
\end{tabular}

Note: *Statistical significance for frequency was analyzed by the Chi-squared test and the continuous variables were analyzed by the $t$-test.

Table 3 Baseline Characteristics According to Hand Grip, Back Muscle Strength, and Normalized Strength in Non-Diabetes and Incident Diabetes

\begin{tabular}{|c|c|c|c|c|c|c|}
\hline \multirow[t]{3}{*}{ Variables } & \multicolumn{3}{|c|}{ Women $(n=13 \mid 3)$} & \multicolumn{3}{|l|}{ Men $(n=1386)$} \\
\hline & \multirow{2}{*}{$\begin{array}{l}\text { Non-Diabetes } \\
(n=887)\end{array}$} & \multirow{2}{*}{$\begin{array}{l}\text { Incident Diabetes } \\
(n=426)\end{array}$} & \multirow[t]{2}{*}{ P-value* } & \multirow{2}{*}{$\begin{array}{l}\text { Non-Diabetes } \\
(n=836)\end{array}$} & \multirow{2}{*}{$\begin{array}{l}\text { Incident Diabetes } \\
(n=550)\end{array}$} & \multirow[t]{2}{*}{ P-value* } \\
\hline & & & & & & \\
\hline \multicolumn{7}{|l|}{ Hand grip strength (HGS) } \\
\hline HGS (kg) & $22.0 \pm 3.9$ & $21.5 \pm 3.9$ & 0.034 & $35.8 \pm 5.6$ & $35.0 \pm 5.6$ & 0.011 \\
\hline BMI-normalized HGS & $0.92 \pm 0.19$ & $0.85 \pm 0.18$ & $<0.001$ & $1.48 \pm 0.26$ & $1.40 \pm 0.26$ & $<0.001$ \\
\hline Weight-normalized HGS & $0.38 \pm 0.07$ & $0.35 \pm 0.07$ & $<0.001$ & $0.52 \pm 0.09$ & $0.50 \pm 0.09$ & $<0.001$ \\
\hline Weight ${ }^{2 / 3}$-normalized HGS & $2.22 \pm 0.62$ & $1.99 \pm 0.56$ & $<0.001$ & $2.6 I \pm 0.67$ & $2.4 I \pm 0.69$ & $<0.001$ \\
\hline WC-normalized HGS & $0.30 \pm 0.06$ & $0.27 \pm 0.05$ & $<0.001$ & $0.44 \pm 0.08$ & $0.4 I \pm 0.07$ & $<0.001$ \\
\hline WHR-normalized HGS & $28.0 \pm 5.3$ & $26.1 \pm 5.1$ & $<0.001$ & $41.9 \pm 7.1$ & $39.8 \pm 6.8$ & $<0.001$ \\
\hline \multicolumn{7}{|l|}{ Back muscle strength (BMS) } \\
\hline BMS (kg) & $43.2 \pm 12.5$ & $42.1 \pm 12.1$ & 0.118 & $84.7 \pm 18.7$ & $83.5 \pm 20.0$ & 0.275 \\
\hline BMI-normalized BMS & $1.80 \pm 0.54$ & $1.66 \pm 0.50$ & $<0.001$ & $3.5 I \pm 0.82$ & $3.33 \pm 0.78$ & $<0.001$ \\
\hline Weight-normalized BMS & $0.74 \pm 0.22$ & $0.69 \pm 0.21$ & $<0.001$ & $1.24 \pm 0.29$ & $1.19 \pm 0.28$ & $<0.001$ \\
\hline Weight $^{2 / 3}$-normalized BMS & $4.34 \pm 1.49$ & $3.89 \pm 1.42$ & $<0.001$ & $6.17 \pm 1.87$ & $5.7 I \pm I .77$ & $<0.001$ \\
\hline WC-normalized BMS & $0.58 \pm 0.17$ & $0.53 \pm 0.16$ & $<0.001$ & $1.04 \pm 0.24$ & $0.98 \pm 0.23$ & $<0.001$ \\
\hline WHR-normalized BMS & $54.9 \pm 16.2$ & $51.1 \pm 14.8$ & $<0.001$ & $99.2 \pm 22.7$ & $94.8 \pm 22.9$ & $<0.001$ \\
\hline
\end{tabular}

Note: *Statistical significance for continuous variables was analyzed by the $t$-test. 


\section{Cox Proportional Hazards Ratios for} Hand Grip Strength and Normalized Hand Grip Strength for Incident Type 2 Diabetes Mellitus by Gender

Table 4 shows the HRs, 95\% CIs, and P-values for each one SD increase in muscle strength and normalized muscle strength adjusted for the confounding factors. In univariate analysis, the HR per one SD increase in HGS and BMI, weight, weight ${ }^{2 / 3}, \mathrm{WC}$, and WHR-normalized HGS was associated with a lower risk of incident T2DM in women and men $(\mathrm{HR}=0.772-0.690, \mathrm{P} \leq 0.002)$. Similar results were seen for BMS. The HR per one SD increase in BMI, weight, weight ${ }^{2 / 3}$, WC, and WHR-normalized HGS was associated with a lower risk of incident T2DM in women and men $(\mathrm{HR}=0.849-0.752, \mathrm{P}<0.001)$. However, HGS was not associated with incident T2DM in both women and men. In multivariate analysis, HGS and BMS were not associated with incident T2DM in both women and men. However, the HR per one SD increase in BMI, weight, weight $^{2 / 3}$, WC, and WHR-normalized HGS and BMS but not weight-normalized BMS was associated with a lower risk of incident T2DM in women (HR $=0.886-0.842$, $\mathrm{P}<0.024)$. In men, the HR per one SD increase in BMI, WC, and WHR-normalized HGS and BMS was only associated with a lower risk of incident T2DM in women $(\mathrm{HR}=0.903-0.877, \mathrm{P}<0.05)$. Muscle strength and normalized muscle strength were not interactive with incident T2DM in gender. When the weight-normalized and other normalized HGS and BMS measurements were compared, the area under the curve (AUC) showed no significant difference. Additionally, muscle strength was grouped into quartiles according to muscle strength by sex. The highest quartile groups of BMI, weight, weight ${ }^{2 / 3}, \mathrm{WC}$, and WHR-normalized HGS and BMS were associated with a lower risk of incident T2DM after adjusting for confounding factors except for weight-normalized BMS in men (Supplementary Table 1).

\section{Cox Proportional Hazard Ratios of Hand Grip, Back Strength, Normalized Muscle Strength for Incident Type 2 Diabetes Mellitus by Menopausal Status}

Table 5 shows the HR of HGS, BMS, and normalized muscle strength for T2DM by menopausal status. In univariate analysis, the HR per one SD increase in HGS and BMI, weight, weight ${ }^{2 / 3}$, WC, and WHR-normalized HGS and BMS was significant, with a lower risk for incident T2DM in pre- and post-menopausal women, except for the HGS of

Table 4 Cox Proportional Hazards Ratios (HRs) for Hand Grip Strength, Back Muscle Strength, and Normalized Strength for Incident Diabetes According to Sex

\begin{tabular}{|c|c|c|c|c|c|}
\hline \multirow[t]{3}{*}{ Variables } & \multicolumn{2}{|l|}{ Women } & \multicolumn{2}{|l|}{ Men } & \multirow[t]{3}{*}{ P§ } \\
\hline & \multicolumn{2}{|l|}{ HR $(95 \% \mathrm{Cl}) \mathrm{P}^{*}$} & \multicolumn{2}{|l|}{ HR $(95 \% \mathrm{Cl}) \mathrm{P}^{*}$} & \\
\hline & Univariate & Multivariate & Univariate & Multivariate & \\
\hline \multicolumn{6}{|c|}{ Hand grip strength (HGS), per I SD increase } \\
\hline HGS $(\mathrm{kg})$ & $0.882(0.800-0.971) 0.011$ & $0.983(0.887-1.089) 0.736$ & $0.871(0.800-0.949) 0.002$ & $0.944(0.863-1.032) 0.204$ & 0.935 \\
\hline BMI-normalized HGS & $0.71 I(0.643-0.787)<0.001$ & $0.860(0.773-0.957) 0.006$ & $0.75 I(0.686-0.82 I)<0.00 I$ & $0.890(0.809-0.978) 0.015$ & 0.914 \\
\hline Weight-normalized HGS & $0.735(0.665-0.812)<0.001$ & $0.886(0.798-0.984) 0.024$ & $0.771(0.707-0.842)<0.001$ & $0.914(0.833-1.002) 0.055$ & 0.885 \\
\hline Weight $^{2 / 3}$-normalized HGS & $0.693(0.622-0.77 I)<0.001$ & $0.848(0.759-0.948) 0.004$ & $0.772(0.704-0.847)<0.001$ & $0.924(0.836-1.020) 0.118$ & 0.406 \\
\hline WC-normalized HGS & $0.690(0.625-0.762)<0.001$ & $0.842(0.755-0.939) 0.002$ & $0.736(0.674-0.804)<0.001$ & $0.877(0.798-0.964) 0.006$ & 0.907 \\
\hline WHR-normalized HGS & $0.728(0.660-0.803)<0.001$ & $0.858(0.771-0.956) 0.005$ & $0.761(0.697-0.83 \mathrm{I})<0.00 \mathrm{I}$ & $0.877(0.799-0.962) 0.005$ & 0.929 \\
\hline \multicolumn{6}{|c|}{ Back muscle strength (BMS), per I SD increase } \\
\hline BMS (kg) & $0.914(0.830-1.006) 0.067$ & $0.960(0.868-1.062) 0.433$ & $0.940(0.864-1.023) 0.155$ & $0.953(0.873-1.04 I) 0.287$ & 0.598 \\
\hline BMI-normalized BMS & $0.783(0.708-0.865)<0.001$ & $0.880(0.792-0.977) 0.017$ & $0.828(0.760-0.90 I)<0.001$ & $0.903(0.826-0.987) 0.024$ & 0.980 \\
\hline Weight-normalized BMS & $0.811(0.734-0.895)<0.001$ & $0.906(0.817-1.005) 0.061$ & $0.849(0.780-0.924)<0.001$ & $0.923(0.846-1.008) 0.076$ & 0.988 \\
\hline Weight ${ }^{2 / 3}$-normalized BMS & $0.752(0.678-0.835)<0.001$ & $0.873(0.784-0.973) 0.014$ & $0.811(0.742-0.887)<0.001$ & $0.917(0.835-1.007) 0.070$ & 0.685 \\
\hline WC-normalized BMS & $0.773(0.699-0.854)<0.001$ & $0.87 \mid(0.783-0.970) 0.012$ & $0.822(0.755-0.895)<0.001$ & $0.899(0.822-0.983) 0.020$ & 0.950 \\
\hline WHR-normalized BMS & $0.801(0.725-0.885)<0.001$ & $0.880(0.791-0.978) 0.017$ & $0.847(0.778-0.922)<0.001$ & $0.903(0.826-0.986) 0.024$ & 0.972 \\
\hline
\end{tabular}

Notes: *Adjusted for age, family history of diabetes, exercise, alcohol consumption ex- and current smoking status, job, HDL-cholesterol, triglycerides, fasting glucose, hypertension, cardiovascular diseases, and energy consumption for men at baseline, and adjusted for menopause for women at baseline in addition to the adjustments made for men. §Indicates $P$ for the interaction of gender*muscle strength. 
Table 5 Cox Proportional Hazards Ratios (HRs) for Hand Grip Strength, Back Muscle Strength, and Normalized Strength for Incident Diabetes in Premenopausal and Postmenopausal Women

\begin{tabular}{|c|c|c|c|c|c|}
\hline \multirow[t]{3}{*}{ Variables } & \multirow{2}{*}{\multicolumn{2}{|c|}{$\begin{array}{l}\text { Premenopausal } \\
\text { HR }(95 \% \mathrm{CI}) \mathbf{P}^{*}\end{array}$}} & \multirow{2}{*}{\multicolumn{2}{|c|}{$\begin{array}{l}\text { Postmenopausal } \\
\text { HR (95\% Cl) P* }\end{array}$}} & \multirow[t]{3}{*}{$\mathbf{P \S}$} \\
\hline & & & & & \\
\hline & Univariate & Multivariate & Univariate & Multivariate & \\
\hline \multicolumn{6}{|c|}{ Hand grip strength (HGS), per I SD increase } \\
\hline HGS (kg) & $0.886(0.794-0.990) 0.032$ & $0.953(0.848-1.069) 0.411$ & $0.949(0.76 I-I .183) 0.639$ & $1.077(0.854-1.357) 0.530$ & 0.646 \\
\hline BMI-normalized HGS & $0.713(0.635-0.800)<0.001$ & $0.840(0.743-0.950) 0.006$ & $0.76 I(0.609-0.95 I) 0.017$ & $0.924(0.726-1.175) 0.518$ & 0.279 \\
\hline Weight-normalized HGS & $0.731(0.652-0.820)<0.001$ & $0.860(0.762-0.972) 0.015$ & $0.799(0.645-0.989) 0.040$ & $0.962(0.766-1.208) 0.736$ & 0.197 \\
\hline Weight $^{2 / 3}$-normalized HGS & $0.684(0.604-0.774)<0.001$ & $0.829(0.728-0.944) 0.005$ & $0.762(0.611-0.949) 0.015$ & $0.908(0.724-1.139) 0.405$ & 0.304 \\
\hline WC-normalized HGS & $0.689(0.615-0.773)<0.001$ & $0.820(0.723-0.929) 0.002$ & $0.747(0.600-0.929) 0.009$ & $0.911(0.711-1.168) 0.464$ & 0.185 \\
\hline WHR-normalized HGS & $0.723(0.646-0.809)<0.001$ & $0.830(0.734-0.938) 0.003$ & $0.800(0.643-0.996) 0.046$ & $0.957(0.752-1.219) 0.724$ & 0.120 \\
\hline \multicolumn{6}{|c|}{ Back muscle strength (BMS), per I SD increase } \\
\hline BMS (kg) & $0.928(0.831-1.036) 0.181$ & $0.954(0.85 \mathrm{I}-1.070) 0.423$ & $0.885(0.720-1.088) 0.247$ & $0.919(0.738-1.145) 0.452$ & 0.642 \\
\hline BMI-normalized BMS & $0.795(0.710-0.891)<0.001$ & $0.878(0.780-0.990) 0.034$ & $0.764(0.614-0.95 I) 0.016$ & $0.84 I(0.667-1.06 I) 0.144$ & 0.655 \\
\hline Weight-normalized BMS & $0.818(0.730-0.916) 0.001$ & $0.900(0.800-1.012) 0.079$ & $0.802(0.648-0.991) 0.041$ & $0.878(0.703-1.095) 0.249$ & 0.536 \\
\hline Weight $^{2 / 3}$-normalized BMS & $0.753(0.669-0.849)<0.001$ & $0.867(0.766-0.98 I) 0.024$ & $0.764(0.612-0.954) 0.018$ & $0.863(0.689-1.082) 0.202$ & 0.522 \\
\hline WC-normalized BMS & $0.784(0.699-0.880)<0.001$ & $0.869(0.770-0.982) 0.024$ & $0.764(0.618-0.944) 0.013$ & $0.832(0.659-1.05 I) 0.122$ & 0.557 \\
\hline WHR-normalized BMS & $0.808(0.721-0.905)<0.001$ & $0.873(0.774-0.984) 0.027$ & $0.798(0.647-0.984) 0.035$ & $0.855(0.680-1.075) 0.180$ & 0.471 \\
\hline
\end{tabular}

Notes: *Adjusted for age, family history of diabetes, exercise, alcohol consumption ex- and current smoking status, job, HDL-cholesterol, triglycerides, fasting glucose, hypertension, cardiovascular diseases, and energy consumption for men at baseline, and adjusted for menopause for women at baseline in addition to the adjustments made for men. §Indicates $P$ for the interaction of menopause status*muscle strength.

postmenopausal women and the BMS of pre- and postmenopausal women $(\mathrm{HR}=0.818-0.684, \mathrm{P}<0.05$ for premenopausal women and $\mathrm{HR}=0.802-0.761, \mathrm{P}<0.05$ for postmenopausal women). In multivariate analysis, the HR per one $\mathrm{SD}$ increase in BMI, weight, weight ${ }^{2 / 3}$, WC, and WHRnormalized HGS and BMS was only significantly associated with incident T2DM in premenopausal women $(\mathrm{HR}=0.878-$ $0.820, \mathrm{P}<0.05$ ). However, postmenopausal women were not associated with incident T2DM. Additionally, muscle strength was grouped into quartiles according to muscle strength by menopausal status. The highest quartile groups of BMI, weight, weight ${ }^{2 / 3}$, WC, and WHR-normalized HGS and BMS were associated with a lower risk for incident T2DM after adjusting for the confounding factors in premenopausal women (Supplementary Table 2). The highest quartile group of BMI in WC-normalized BMS was only associated with a lower risk for incident T2DM after adjusting for confounding factors in postmenopausal women. Muscle strength and normalized muscle strength were not interactive with incident T2DM in menopausal status. For sensitivity analyses, we calculated the HR, 95\% CI, and P-value of normalized HGS and BMS for incident T2DM during the second, sixth, and ninth follow-up (Supplementary Table 3). We were able to confirm that a similar trend was maintained during the followup period.

\section{Discussion}

The results of this prospective population-based study were similar to those of previous studies. Additionally, we showed three new results. First, we revealed that WC and WHR-normalized HGS and BMS were related to incident T2DM. Second, normalized BMS was associated with incident T2DM. Third, normalized HGS and BMS were associated with incident T2DM only in premenopausal women.

The loss of muscle mass and muscle strength that occurs with age is widely regarded as one of the major causes of chronic diseases and weakness with aging. ${ }^{28}$ Observational studies have shown that muscle mass and strength peak before age 40 and then steadily decline with aging. ${ }^{29}$ Skeletal muscles, which account for $40 \%$ of the total weight, deteriorate quantitatively and qualitatively with aging. ${ }^{30}$ It has been reported that the prevalence of metabolic diseases due to decreased muscle strength increases with age, ${ }^{31,32}$ and falls and fractures, mobility impairments, complications, infections, metabolic disorders, and mortality increase as muscle strength decreases. $^{33-35}$

Several studies reported associations between T2DM and HGS. ${ }^{6-8}$ However, there is also evidence that HGS is not associated with T2DM. $^{9-11}$ Consequentially, the 
relationship between T2DM and HGS is controversial. However, in a meta-analysis of 10 observational cohort studies, the pooled fully-adjusted relative risk of incident T2DM in the higher tertile of HGS values had a 0.73 -fold lower risk. ${ }^{36}$

The contradictory evidence could be due to heterogeneity in the criteria that were used between the studies when defining weak muscle strength (absolute or normalized muscle strength). Normalized muscle strength has been proposed as a solution to these problems. ${ }^{20} \mathrm{HSG}$ or BMS is related to factors such as weight, BMI, and waist and can act as a confounding variable, so it is recommended to use a normalized method when investigating the relationship with disease. ${ }^{20}$ It can be said that the normalized results of various body variables such as BMI, weight, and waist are more reliable and accurate. Several studies reported associations between T2DM and weight $^{37,38}$ and weight ${ }^{2 / 3} 39$-normalized HSG.

Abdominal obesity, which is approximated by WC and the WHR is typically seen in overweight and obese people. $^{23}$ Abdominal obesity is highly correlated with BMI and associated with the incidence of T2DM. ${ }^{40}$ Reductions in muscle mass are positively correlated with central obesity and can increase the risk of T2DM development. ${ }^{41}$ Moreover, WC is strongly correlated with T2DM, which is a better indicator for T2DM than the BMI. $^{42}$ In this study, we showed that WC and WHRnormalized HGS and BMS were associated with incident T2DM in both males and females in this study. WC and WHR-normalized muscle strength are also considered important in predicting T2DM risk.

BMS refers to the deep muscles at the center of the body, and are representative core muscles. It has been reported that BMS decreases by $50 \%$ between the ages of 30 to 60 years, and the cross-sectional area of the body's central muscles decreases as the fat expansion rate increases with age. ${ }^{43}$ As BMS weakens, insulin resistance appears, and BMS is significantly correlated with HGS and body muscle mass. ${ }^{21}$ This is thought to be an important cause of the gradual acceleration of the incidence of T2DM. ${ }^{22}$ In this study, we showed that WC and WHR-normalized BMS were associated with incident T2DM in both males and females in this study. BMI, weight, weight ${ }^{2 / 3}$, WC, and WHR-normalized BMS showed similar results for incident T2DM. Therefore, BMS was also considered important in predicting T2DM risk.

In general, postmenopausal women have a higher incidence of T2DM than premenopausal women. ${ }^{44}$ However, in this study, the association between normalized HGS and BMS and incident T2DM was higher in premenopausal women. A recent study reported that the skeletal muscle mass index (SMI)/weight of premenopausal women represented a 2-fold higher risk for incident T2DM than that of menopause women. ${ }^{45}$ This suggests that muscle strength had a greater effect on premenopausal than postmenopausal women. Menopausal status is associated with an increase in body fat mass and central obesity and a decrease in muscle mass and strength. $^{46,47}$ Visceral adiposity could demonstrate a more sensitive index of metabolic risk when combined with measurements of low muscle mass. ${ }^{48}$ In a combined study of muscle mass and fat mass, low muscle/high fat and high muscle/high fat were associated with higher fasting glucose and HOMA-IR levels compared to the low muscle/low fat and high muscle/low fat group. ${ }^{49}$ These results suggest that the relationship between body muscle and fat mass is significantly related to glucose metabolism and insulin resistance. However, there has been no study on the relationship between mass or muscle strength and fat in premenopausal and postmenopausal women, so more research is needed.

This study had some limitations. First, the number of postmenopausal women in the quartile groups was small. Second, the study population was limited to Koreans and because of racial differences, it will be difficult to generalize the research results. Third, muscle mass, muscle strength, and function may be different in various outcomes, so more research on this is needed.

\section{Conclusion}

The results of this study showed that normalized HGS and BMS were more sensitive indexes for incident T2DM than simple HGS and BSM. As this study was limited to Koreans, it will be difficult to generalize the results as there will be differences between races. Therefore, age and sex-specific normative HGS and BMS data are needed to establish the cutoffs values for low HGS and BMS in ethnic populations. HGS and BMS will be conveniently used as the simplest predictive method for T2DM incidence to screen subjects with T2DM risk.

\section{Ethical Committee Permission}

This study was approved by the Institutional Review Board of the Korea University Ansan Hospital, and written informed consent was provided by all study subjects, and was conducted in accordance with the Declaration of Helsinki. 


\section{Acknowledgment}

This research was supported by the Bio \& Medical Technology Development Program of the National Research Foundation of Korea (NRF) funded by the Ministry of Science, ICT, \& Future Planning (no. 2015M3A9B6027142), by research funding (nos. 2001347-6111-221, 2002-347-6111-221, 2003-347-6111-221, 2004-E71001-00， 2005-E71001-00， 2006-E71005-00, 2007-E71001-00， 2008-E71001-00， 2009-E71002-00, 2010-E71001-00, 2011-E71004-00, 2012-E71005-00, 2013-E71005-00， 2014-E71003-00， 2015-P71001-00, 2016-E71003-00, 2017-E71001-00, and 2018-E7101-00) from the Korea Centers for Disease Control and Prevention, and by a Korea University Grant.

\section{Disclosure}

The authors declare that there are no conflicts of interest regarding the publication of this article.

\section{References}

1. Li G, Zhang P, Wang J, et al. The long-term effect of lifestyle interventions to prevent diabetes in the China Da Qing Diabetes Prevention Study: a 20-year follow-up study. Lancet. 2008;371 (9626):1783-1789. doi:10.1016/S0140-6736(08)60766-7

2. American Diabetes A. Standards of medical care in diabetes-2010. Diabetes Care. 2010;33(Suppl 1):S11-S61. doi:10.2337/dc10-S011

3. Zhang P, Zhang X, Brown J, et al. Global healthcare expenditure on diabetes for 2010 and 2030. Diabetes Res Clin Pract. 2010;87 (3):293-301. doi:10.1016/j.diabres.2010.01.026

4. Bohannon RW. Grip strength: an indispensable biomarker for older adults. Clin Interv Aging. 2019;14:1681-1691. doi:10.2147/CIA S194543

5. Wall BT, Dirks ML, van Loon LJ. Skeletal muscle atrophy during short-term disuse: implications for age-related sarcopenia. Ageing Res Rev. 2013;12(4):898-906. doi:10.1016/j.arr.2013.07.003

6. Kim CR, Jeon Y-J, Jeong T, Mogi M. Risk factors associated with low handgrip strength in the older Korean population. PLoS One. 2019;14(3):e0214612. doi:10.1371/journal.pone.0214612

7. Larsen BA, Wassel CL, Kritchevsky SB, et al. Association of muscle mass, area, and strength with incident diabetes in older adults: the Health ABC Study. J Clin Endocrinol Metab. 2016;101 (4):1847-1855. doi:10.1210/jc.2015-3643

8. Cuthbertson DJ, Bell JA, Ng SY, Kemp GJ, Kivimaki M, Hamer M. Dynapenic obesity and the risk of incident type 2 diabetes: the English Longitudinal Study of ageing. Diabet Med. 2016;33 (8):1052-1059. doi:10.1111/dme.12991

9. Karvonen-Gutierrez CA, Peng Q, Peterson M, Duchowny K, Nan B, Harlow S. Low grip strength predicts incident diabetes among midlife women: the Michigan Study of women's health across the nation. Age Ageing. 2018;47(5):685-691. doi:10.1093/ageing/afy067

10. Giglio BM, Mota JF, Wall BT, Pimentel GD. Low handgrip strength is not associated with type 2 diabetes mellitus and hyperglycemia: a Population-Based Study. Clin Nutr Res. 2018;7(2):112-116. doi:10.7762/cnr.2018.7.2.112

11. Leong DP, Teo KK, Rangarajan S, et al. Prognostic value of grip strength: findings from the Prospective Urban Rural Epidemiology (PURE) study. Lancet. 2015;386(9990):266-273. doi:10.1016/S01406736(14)62000-6
12. Looker AC, Wang CY. Prevalence of reduced muscle strength in older U.S. adults: United States, 2011-2012. NCHS Data Brief. 2015;179:1-8.

13. Pattison RJ, Catalá C. Evaluating auxin distribution in tomato (Solanum lycopersicum) through an analysis of the PIN and AUX/ LAX gene families. Plant J. 2012;70(4):585-598. doi:10.1111/ j.1365-313X.2011.04895.x

14. Gallagher D, Visser M, De Meersman RE, et al. Appendicular skeletal muscle mass: effects of age, gender, and ethnicity. $J \mathrm{Appl}$ Physiol (1985). 1997;83(1):229-239. doi:10.1152/jappl.1997.8 3.1 .229

15. Slemenda C, Heilman DK, Brandt KD, et al. Reduced quadriceps strength relative to body weight: a risk factor for knee osteoarthritis in women? Arthritis Rheum. 1998;41(11):1951-1959. doi:10.1002/ 1529-0131(199811)41:11<1951::AID-ART9>3.0.CO;2-9

16. Th IJ, Schaper NC, Melai T, Meijer K, Willems PJ, Savelberg HH. Lower extremity muscle strength is reduced in people with type 2 diabetes, with and without polyneuropathy, and is associated with impaired mobility and reduced quality of life. Diabetes Res Clin Pract. 2012;95(3):345-351. doi:10.1016/j.diabres.2011.10.026

17. Cawthon PM, Travison TG, Manini TM, et al. Establishing the link between lean mass and grip strength cut points with mobility disability and other health outcomes: proceedings of the sarcopenia definition and outcomes consortium conference. J Gerontol a Biol Sci Med Sci. 2020;75(7):1317-1323. doi:10.1093/gerona/glz081

18. Maranhao Neto GA, Oliveira AJ, Pedreiro RC, et al. Normalizing handgrip strength in older adults: an allometric approach. Arch Gerontol Geriatr. 2017;70:230-234. doi:10.1016/j.archger.20 17.02.007

19. Crewther BT, Gill N, Weatherby RP, Lowe T. A comparison of ratio and allometric scaling methods for normalizing power and strength in elite rugby union players. J Sports Sci. 2009;27(14):1575-1580. doi:10.1080/02640410903348657

20. Choquette S, Bouchard DR, Doyon CY, Senechal M, Brochu M, Dionne IJ. Relative strength as a determinant of mobility in elders 67-84 years of age. a nuage study: nutrition as a determinant of successful aging. $J$ Nutr Health Aging. 2010;14(3):190-195. doi:10.1007/s12603-010-0047-4

21. Toyoda H, Hoshino M, Ohyama S, et al. The association of back muscle strength and sarcopenia-related parameters in the patients with spinal disorders. Eur Spine J. 2019;28(2):241-249. doi:10.1007/s00586-018-5858-8

22. Grontved A, Ried-Larsen M, Ekelund U, Froberg K, Brage S, Andersen LB. Independent and combined association of muscle strength and cardiorespiratory fitness in youth with insulin resistance and beta-cell function in young adulthood: the European Youth Heart Study. Diabetes Care. 2013;36(9):2575-2581. doi:10.2337/dc122252

23. Vazquez G, Duval S, Jacobs DR Jr, Silventoinen K. Comparison of body mass index, waist circumference, and waist/hip ratio in predicting incident diabetes: a meta-analysis. Epidemiol Rev. 2007;29:115-128. doi:10.1093/epirev/mxm008

24. Chalmers J, MacMahon S, Mancia G, et al. 1999 World Health Organization-international society of hypertension guidelines for the management of hypertension. Guidelines sub-committee of the World Health Organization. Clin Exp Hypertens. 1999;21(5-6):1009-1060. doi:10.3109/10641969909061028

25. Alberti KG, Zimmet PZ. Definition, diagnosis and classification of diabetes mellitus and its complications. Part 1: diagnosis and classification of diabetes mellitus provisional report of a WHO consultation. Diabet Med. 1998;15(7):539-553. doi:10.1002/(SICI) 1096-9136(199807)15:7<539::AID-DIA668>3.0.CO;2-S

26. Wilson PW, Meigs JB, Sullivan L, Fox CS, Nathan DM, D'Agostino RB Sr. Prediction of incident diabetes mellitus in middle-aged adults: the Framingham Offspring Study. Arch Intern Med. 2007;167(10):1068-1074. doi:10.1001/archinte.167.10.1068 
27. Carnethon MR, Kinder LS, Fair JM, Stafford RS, Fortmann SP. Symptoms of depression as a risk factor for incident diabetes: findings from the National Health and Nutrition Examination Epidemiologic Follow-up Study, 1971-1992. Am J Epidemiol. 2003;158(5):416-423. doi:10.1093/aje/kwg172

28. Fried LP, Guralnik JM. Disability in older adults: evidence regarding significance, etiology, and risk. J Am Geriatr Soc. 1997;45 (1):92-100. doi:10.1111/j.1532-5415.1997.tb00986.x

29. Frontera WR, Hughes VA, Lutz KJ, Evans WJ. A cross-sectional study of muscle strength and mass in 45- to 78-yr-old men and women. J Appl Physiol (1985). 1991;71(2):644-650. doi:10.1152/ jappl.1991.71.2.644

30. Kim KM, Jang HC, Lim S. Differences among skeletal muscle mass indices derived from height-, weight-, and body mass index-adjusted models in assessing sarcopenia. Korean J Intern Med. 2016;31 (4):643-650. doi:10.3904/kjim.2016.015

31. Wang T, Feng X, Zhou J, et al. Type 2 diabetes mellitus is associated with increased risks of sarcopenia and pre-sarcopenia in Chinese elderly. Sci Rep. 2016;6(1):38937. doi:10.1038/srep38937

32. Lauretani F, Russo CR, Bandinelli S, et al. Age-associated changes in skeletal muscles and their effect on mobility: an operational diagnosis of sarcopenia. J Appl Physiol (1985). 2003;95(5):1851-1860. doi:10.1152/japplphysiol.00246.2003

33. Joglekar S, Nau PN, Mezhir JJ. The impact of sarcopenia on survival and complications in surgical oncology: a review of the current literature-author response. J Surg Oncol. 2015;112(8):910. doi:10.1002/jso. 24095

34. Tarantino U, Piccirilli E, Fantini M, Baldi J, Gasbarra E, Bei R. Sarcopenia and fragility fractures: molecular and clinical evidence of the bone-muscle interaction. J Bone Joint Surg Am. 2015;97 (5):429-437. doi:10.2106/JBJS.N.00648

35. Filippin LI, Teixeira VN, da Silva MP, Miraglia F, da Silva FS. Sarcopenia: a predictor of mortality and the need for early diagnosis and intervention. Aging Clin Exp Res. 2015;27(3):249-254. doi:10.1007/s40520-014-0281-4

36. Kunutsor SK, Isiozor NM, Khan H, Laukkanen JA. Handgrip strength-a risk indicator for type 2 diabetes: systematic review and meta-analysis of observational cohort studies. Diabetes Metab Res Rev. 2020;e3365. doi:10.1002/dmrr.3365

37. Momma H, Sawada SS, Kato K, et al. Physical fitness tests and type 2 diabetes among japanese: a Longitudinal Study from the Niigata Wellness Study. J Epidemiol. 2019;29(4):139-146. doi:10.2188/jea. JE20170280

38. Lee MR, Jung SM, Bang H, Kim HS, Kim YB. Association between muscle strength and type 2 diabetes mellitus in adults in Korea: data from the Korea national health and nutrition examination survey (KNHANES) VI. Medicine (Baltimore). 2018;97(23):e10984. doi:10.1097/MD.0000000000010984
39. Kunutsor SK, Voutilainen A, Laukkanen JA. Handgrip strength improves prediction of type 2 diabetes: a prospective cohort study. Ann Med. 2020;52(8):471-478. doi:10.1080/07853890.2020.1815078

40. Yang XY, Zhang M, Luo XP, et al. Body mass index, waist circumference and waist-to-height ratio associated with the incidence of type 2 diabetes mellitus: a cohort study. Zhonghua Yu Fang Yi Xue Za Zhi. 2016;50(4):328-333. doi:10.3760/cma.j.issn.02539624.2016.04.009

41. de Carvalho DHT, Scholes S, Santos JLF, de Oliveira C, Alexandre TDS. Does abdominal obesity accelerate muscle strength decline in older adults? Evidence from the English Longitudinal Study of ageing. J Gerontol a Biol Sci Med Sci. 2019;74 (7):1105-1111. doi:10.1093/gerona/gly178

42. Wang Y, Rimm EB, Stampfer MJ, Willett WC, Hu FB. Comparison of abdominal adiposity and overall obesity in predicting risk of type 2 diabetes among men. Am J Clin Nutr. 2005;81(3):555-563. doi:10.1093/ajcn/81.3.555

43. Takayama K, Kita T, Nakamura H, et al. New predictive index for lumbar paraspinal muscle degeneration associated with aging. Spine (Phila Pa 1976). 2016;41(2):E84-E90. doi:10.1097/BRS.000000 0000001154

44. Heianza Y, Arase Y, Kodama S, et al. Effect of postmenopausal status and age at menopause on type 2 diabetes and prediabetes in Japanese individuals: Toranomon Hospital Health Management Center Study 17 (TOPICS 17). Diabetes Care. 2013;36(12):4007-4014. doi: $10.2337 /$ dc13-1048

45. Hong S, Chang Y, Jung HS, Yun KE, Shin H, Ryu S. Relative muscle mass and the risk of incident type 2 diabetes: a cohort study. PLoS One. 2017;12(11):e0188650. doi:10.1371/journal.pone.0188650

46. Maltais ML, Desroches J, Dionne IJ. Changes in muscle mass and strength after menopause. J Musculoskelet Neuronal Interact. 2009;9 (4):186-197.

47. Toth MJ, Tchernof A, Sites CK, Poehlman ET. Effect of menopausal status on body composition and abdominal fat distribution. Int J Obes Relat Metab Disord. 2000;24(2):226-231. doi:10.1038/sj.ijo.0801118

48. Lim KI, Yang SJ, Kim TN, et al. The association between the ratio of visceral fat to thigh muscle area and metabolic syndrome: the Korean Sarcopenic Obesity Study (KSOS). Clin Endocrinol (Oxf). 2010;73 (5):588-594. doi:10.1111/j.1365-2265.2010.03841.x

49. Kim K, Park SM. Association of muscle mass and fat mass with insulin resistance and the prevalence of metabolic syndrome in Korean adults: a cross-sectional study. Sci Rep. 2018;8(1):2703. doi:10.1038/s41598-018-21168-5

\section{Publish your work in this journal}

Diabetes, Metabolic Syndrome and Obesity: Targets and Therapy is an international, peer-reviewed open-access journal committed to the rapid publication of the latest laboratory and clinical findings in the fields of diabetes, metabolic syndrome and obesity research. Original research, review, case reports, hypothesis formation, expert opinion and commentaries are all considered for publication. The manuscript management system is completely online and includes a very quick and fair peer-review system, which is all easy to use. Visit http://www.dovepress.com/testimonials.php to read real quotes from published authors. 